\title{
Densidad y estatus poblacional de monos aulladores rojos en un guadual, fragmento aislado, La Tebaida, Quindío, Colombia
}

\author{
Carolina Gómez- Posada ${ }^{1 *}$, Zulima Álvarez ${ }^{2}$, Paola Giraldo-Chavarriaga ${ }^{3}$ \\ ${ }^{1}$ Fundación EcoAndina / Wildlife Conservation Society Programa Colombia, Cali. Dirección actual: \\ Department of Biology, University of Washington, 24 Kincaid Hall, Seattle, WA 98195, USA \\ ${ }^{2}$ Grupo de Estudios en Manejo de Vida Silvestre y Conservación (GEMAVIC), Museo de Historia Natural, \\ Universidad del Cauca, Carrera 2 No 1 - 25 Popayán, Colombia \\ ${ }^{3}$ Departamento de Biología, Universidad del Valle, calle 13 \# 100 - 00, Cali, Colombia \\ *carogomezposada@hotmail.com,cgomez@u.washington.edu
}

Recibido: 25-09-2008; Aceptado: 27-08-2009

\begin{abstract}
Resumen
Gran parte de los remanentes boscosos alrededor de $1000 \mathrm{~m}$ de altitud en el eje cafetero Colombiano, son guaduales, bosques con predominio de bambú espinoso Guauda spp. Estos remanentes sostienen las últimas poblaciones de monos aulladores rojos en la franja entre 800 y $1300 \mathrm{~m}$ de altitud en la Cordillera Central Colombiana. Objetivo: Evaluar el estado poblacional de aulladores en un fragmento de guadua de 51,4 ha a $1100 \mathrm{~m}$ en el Quindío. Métodos y resultados: Se estimó por conteo directo una densidad ecológica de 163,4 individuos y 16,9 grupos $/ \mathrm{km}^{2}$, la cual es superior a la normalmente reportada para la especie en bosques continuos, pero similar a la estimada en fragmentos aislados de bosques de montaña. A pesar de la alta densidad, los grupos presentaron un tamaño y composición similar a los registrados para la especie, con mayor número de individuos inmaduros que de hembras adultas $(1,13: 1)$ sugiriendo que la población se encuentra en condiciones estables. Se estimó una abundancia de solo 84 aulladores en el fragmento. El guadual, angosto y alargado, se encuentra aislado entre sistemas productivos y es poco diverso. Sin embargo, la guadua ofrece la estructura necesaria para el desplazamiento y refugio de los aulladores. Conclusión: Estos resultados demuestran la tolerancia de esta especie a la fragmentación y su flexibilidad para persistir en ambientes alterados. Sin embargo, la alta densidad de individuos, baja diversidad vegetal, aislamiento del bosque, efecto de borde y estrecho contacto con sistemas productivos antrópicos son posibles amenazas para la persistencia de esta población.
\end{abstract}

Palabras clave: Alouatta seniculus, bosque premontano, densidad y tamaño de grupos, Guadua spp, Valle de Maravélez

\begin{abstract}
Density and population status of red howler monkeys in an isolated bamboo forest fragment in La Tebaida, Quindío, Colombia. The majority of forest remnants in the coffee growing region in Colombia are guaduales, forests with a predominance of thorny bamboos (Guadua spp.). These remnants support the last remaining populations of red howler monkeys found between 800 and $1,300 \mathrm{~m}$ of altitude in the Central Cordillera of Colombia. Objective: To assess the population status of howler monkeys in a 51.4 ha-bamboo forest fragment at $1,100 \mathrm{~m}$ of altitude. Methods and results: The estimated howler population density in the fragment, based on direct counts, was of 163.4 individuals and 16.9 groups per $\mathrm{km}^{2}$, which is higher than that commonly reported for this species in continuous forests, although similar to the estimated density in isolated fragments of mountain forests. In spite of the high density, the group size and composition are similar to those usually reported for this species, with more immature individuals than female adults (1.13:1). This finding suggests that the population is under a stable condition. We estimated an abundance of just 84 howlers in the fragment. The guadual, narrow and elongated, is isolated by surrounding productive systems and has a low plant diversity. Nevertheless, guadua plants provide a suitable structure for the displacement and shelter of howlers. Conclusion: Our results demonstrate the tolerance of this species to the fragmentation and its plasticity
\end{abstract}


in persisting in altered environments. However, the high density of individuals, low plant diversity, forest isolation, edge effect, and close contact with anthropic productive systems, are all possible threats to the persistence of this population.

Key words: Alouatta seniculus, group size and density, Guadua spp., Maravélez valley, pre-montane forest

\begin{abstract}
Resumo
Densidade e estado populacional de macacos guariba vermelho num bambusal, fragmento isolado, "La Tebaida, Quindío", Colômbia. Grande parte dos remanentes de floresta próximos a 1000m de altitude na região cafeteira da Colômbia são bambusais, florestas com predomínio de bambu espinhoso Guauda spp. Estes remanentes mantêm as últimas populações de macacos guariba vermelho entre 800 e $1300 \mathrm{~m}$ de altitude na Cordilheira Central Colombiana. Objetivo: Avaliar o estado populacional de guaribas num fragmento de bambu de 51,4ha a 1100m em Quindío. Métodos e resultados: Estimou-se por contagem direta uma densidade populacional de 163,4 indivíduos e 16,9 grupos $/ \mathrm{km}^{2}$, a qual é superior à reportada normalmente para a espécie em florestas contínuas, mas semelhante à estimada em fragmentos isolados de floresta de montanha. Apesar da alta densidade, os grupos apresentaram tamanhos e composição similar a aqueles registrados para a espécie, com maior número de indivíduos imaturos do que de fêmeas adultas (1,13:1) sugerindo que a população está em condições estáveis. Estimou-se a abundância em apenas 84 guaribas no fragmento. O bambusal, estreito e alongado, encontra-se isolado entre sistemas produtivos e é pouco diverso. No entanto, o bambu fornece a estrutura necessária para o deslocamento e refúgio para os guaribas. Conclusão: Estes resultados demonstram a tolerância desta espécie à fragmentação e à sua flexibilidade para persistir em ambientes perturbados. No entanto, a alta densidade de indivíduos, a baixa diversidade de plantas, o isolamento da floresta, o efeito de borda e o estreito contacto com sistemas produtivos antrópicos são possíveis ameaças para a persistência dessa população.
\end{abstract}

Palavras chave: Alouatta seniculus, floresta pré-montana, densidade e tamanho dos grupos, Guadua spp, Valle de Maravélez

\section{Introducción}

Debido a los procesos de apropiación y extracción de material vegetal para madera, cultivos o potreros para el ganado, en la región Andina Colombiana se han deteriorado drásticamente los bosques y por consiguiente, la fauna que en ellos habita $(1,2)$. La franja de vegetación de bosque premontano que se extiende aproximadamente entre 900 y $1800 \mathrm{~m}$ de altitud, es una de las más afectadas puesto que sostiene gran cantidad de asentamientos humanos y zonas agrícolas (como por ejemplo las dedicadas al café). Muchos de los remanentes boscosos en esta zona de vida son corredores angostos que protegen el curso de quebradas y ríos, o se encuentran en lugares con pendientes muy pronunciadas. En los remanentes premontanos situados el eje cafetero colombiano (cordillera Central), predomina la guadua, el bambú nativo de mayor importancia en el país. La guadua es un bambú leñoso y espinoso (Poacecae, subfamilia Bambusoideae) con más de 70 especies en Colombia y Guadua angustifolia es una de las más abundantes en las montañas colombianas. En la zona andina se estima que existen alrededor de 36.000 ha de guaduales, de las cuales el $95 \%$ son naturales. En estos fragmentos la guadua prevalece en todos los estratos del bosque y pocos árboles crecen dispersos entre éstas $(3,4)$.

En la región del eje cafetero, una de las pocas especies de primates que aún persiste es el mono aullador rojo (Alouatta seniculus). Esta es una especie de amplia distribución en Colombia y no es considerada en peligro de extinción. Sin embargo, las poblaciones andinas, especialmente las de bosques premontanos, podrían ser vulnerables por la destrucción de sus hábitat $(5,6)$. Existe muy poca información acerca de la biología de estos monos en bosques andinos y de cómo se encuentran sus poblaciones en esta región altamente intervenida.

En el departamento del Quindío, municipio de La Tebaida, se encuentra un rodal de guadua en el Valle de Maravélez, donde habita una población de monos aulladores rojos. En el presente trabajo se evaluó la densidad de monos aulladores en este guadual, el tamaño y la composición de grupos y se analizó el estado de dicha población. Este estudio arroja información sobre el estado actual de las poblaciones de aulladores rojos en esta zona del país y hace parte de un estudio a largo plazo que está documentando la respuesta de esta especie a la fragmentación y destrucción de sus hábitat (6-8).

\section{Materiales y métodos}

\section{Área de estudio}

El fragmento de bosque estudiado es un rodal de guadua que se encuentra en las veredas Maravélez, Palogrande y La Argentina, municipio de La Tebaida, departamento del Quindío (Figura 1). Se localiza en la vertiente occidental de la cordillera Central $\left(4^{\circ} 24^{\prime} \mathrm{N}, 75^{\circ} 47^{\prime} \mathrm{W}\right)$, en predios de al menos nueve fincas de propiedad privada. El bosque se 
localiza entre $1000 \mathrm{~m}$ y $1200 \mathrm{~m}$ de altitud, con una temperatura que oscila entre 18 y $24{ }^{\circ} \mathrm{C}$; presenta un clima medio y húmedo transicional a medio seco (9). El régimen de precipitación es bimodal, con dos períodos lluviosos de marzo a mayo y de octubre a diciembre, y un promedio anual de precipitación de $1800 \mathrm{~mm}$ (datos suministrados por CVC estaciones metereológicas La Camelia y Miravalles, desde 1947 y 1969). El bosque original debió ser premontano húmedo, según Holdridge (9).

El rodal de guadua estudiado es un angosto corredor de bosque de galería que sigue el curso de la quebrada Palonegro, hasta su desembocadura en la quebrada Cristales (altamente contaminada por las descargas de fincas cafeteras), afluente del río Quindío (Figura 1). El rodal es una franja más o menos continua, inmersa en una matriz de cultivos de cítricos, maíz, plátano y de potreros para ganadería. Algunos de los rodales están protegidos voluntariamente por los propietarios; principalmente para evitar erosión y caída del talud de la quebrada. La mayoría presenta entresaca de guadua para uso doméstico. En este bosque se han liberado diferentes especies de fauna silvestre decomisadas por entidades ambientales.

\section{Historia de uso y conectividad, tamaño del bosque}

Fueron analizadas fotografías aéreas de la zona, correspondientes a diferentes años (SIG), para estimar cómo ha sido la pérdida o la recuperación de la cobertura boscosa durante los últimos años. Adicionalmente se obtuvo información a partir de las observaciones realizadas durante el trabajo de campo, entrevistas informales a los propietarios, trabajadores de las fincas y funcionarios de entidades ambientales de la región, acerca de la historia del uso del bosque, de la historia de la zona en general y de las actividades realizadas en las zonas aledañas. Se revisaron documentos como el POT (plan de ordenamiento territorial) del municipio y literatura adicional sobre la colonización de la zona estudiada.

\section{Estimación de la densidad de aulladores}

Para estimar la densidad de monos se realizó un conteo directo de los individuos y grupos en una zona del guadual (Figura 1). La zona evaluada fue delimitada de acuerdo a

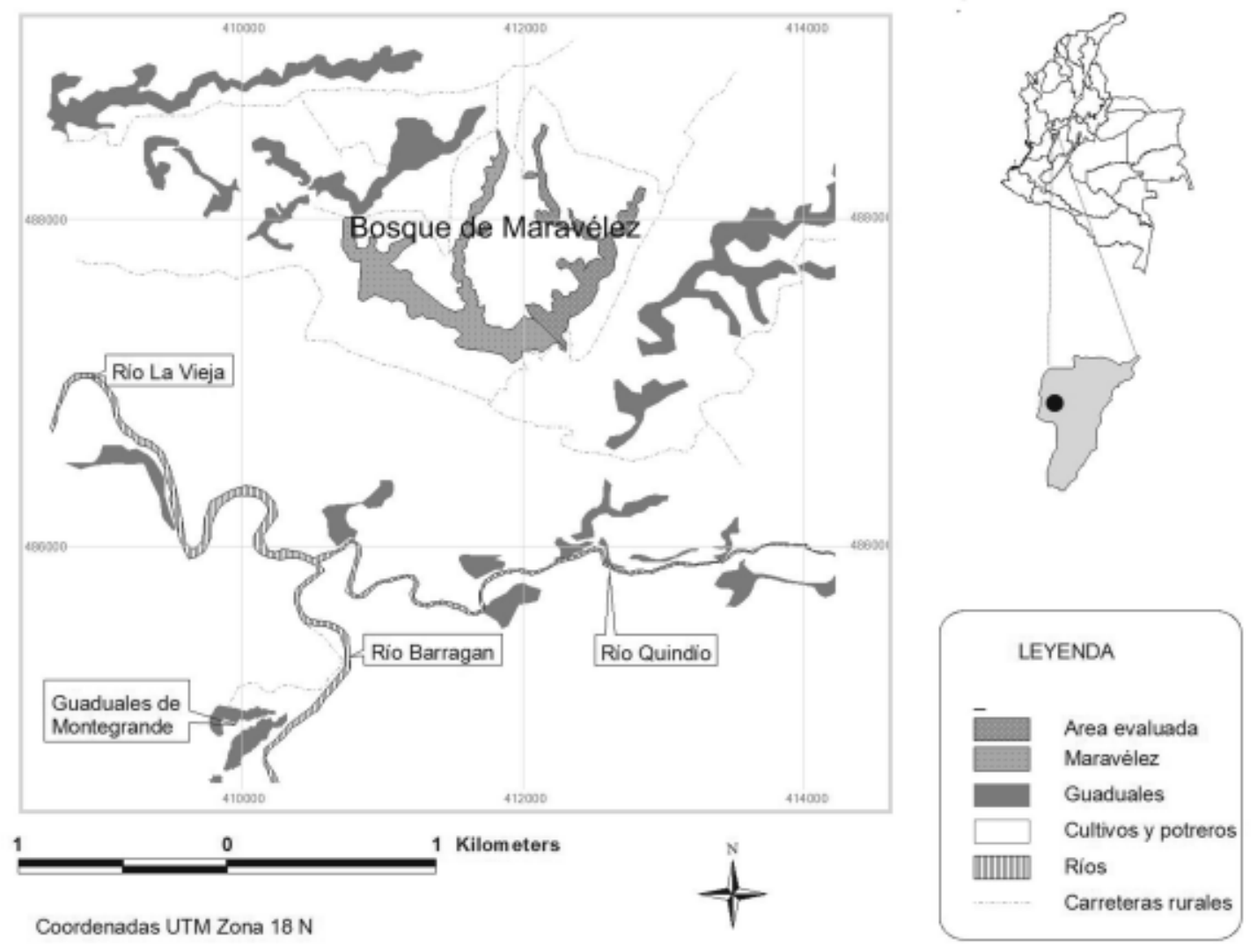

Figura 1. Bosque de Maravélez, Municipio de La Tebaida, Quindío, Colombia. 
la factibilidad del terreno para desarrollar el trabajo (la zona es muy pendiente y presenta áreas de difícil acceso). Se definió e identificó el número de individuos y el número de grupos de aulladores presentes. La densidad de población fue calculada como densidad ecológica: el número de individuos dividido por el área disponible o muestreada (10). Para estimar el tamaño de la población de aulladores en el Bosque de Maravélez, fue utilizada la densidad calculada de individuos y extrapolada al tamaño total del fragmento.

\section{Estructura y composición de los grupos}

Durante el conteo de grupos se registró el número de individuos en cada tropa, especificando edad y sexo $(10,11)$ : adulto, subadulto, juvenil e infante. Las tropas fueron diferenciadas por características como el tamaño, la composición, las características físicas de algunas individuos (color, cicatrices, entre otras) y la consistencia en la localización espacial.

\section{Determinación de la composición y estructura vegetal}

Fue evaluada la disponibilidad de recursos estructurales y alimenticios para los aulladores. Con este fin, se midieron algunas variables teniendo en cuenta los requerimientos de este género de primates $(10,12-14)$ en seis parcelas de vegetación ( 50 x $4 \mathrm{~m}$ ) ubicadas aleatoriamente en la zona evaluada. En las parcelas se tomaron registros del DAP, altura, área de la copa, porcentaje de cobertura del dosel y especie de los árboles con DAP > $10 \mathrm{~cm}$.

\section{Resultados}

\section{Historia de uso y conectividad, tamaño del bosque}

El rodal de guadua estudiado es un fragmento alargado y angosto de 51,4 ha en forma de J (Figura 1). Presenta varios ramales, igualmente angostos pero más cortos, que desembocan al eje principal. La relación área : perímetro es de 1:26.

La fotografía más antigua que se halló de la zona, es del año 1959. En dicho año, ya se observaba la mancha de guadal con la forma actual. Sin embargo, existían otros guaduales e incluso parches de bosque a menos de $100 \mathrm{~m}$. Adicionalmente, cultivos de café con sombrío, mantenían en ese período la conexión entre fragmentos. El área com- pleta del guadual era de 45,3 ha. En la fotografía de 1977 ya habían desaparecido los parches cercanos de bosques, y el guadual lucía similar al actual. Es posible que la cercanía con otros guaduales durante la década de los 60`s le permitiera a los aulladores desplazarse entre éstos. Hacia el final de los 70's, cuando desaparecieron los bosques de los alrededores, la población de aulladores debió quedar aislada. Para el año 2005 (en el transcurso de 46 años), el guadual ganó 6,1 ha de tamaño, pero aumentó el aislamiento con otros guaduales. Este incremento en área se debió principalmente a las zonas más pendientes, ya que por problemas erosivos los propietarios dejaron regenerar las zonas con pendientes muy pronunciadas.

Actualmente el rodal se encuentra aislado en toda su extensión. El fragmento más cercano esta localizado al costado occidental a $280 \mathrm{~m}$ de distancia, cruzando la carretera rural (Figura 1). La población de aulladores más cercana se encuentra hacia el sur occidente, cruzando el río La Vieja, a 2,5 km de distancia, en los rodales de Montegrande (municipio de Caicedonia, Valle).

No se hallaron registros fotográficos del período en que este fragmento quedó aislado. Por tanto, se revisaron documentos acerca de la historia de colonización de la zona. El municipio de La Tebaida fue fundado en 1914. Sin embargo su fundación fue precedida por el asentamiento de guaqueros y colonos en el inicio del siglo XX. Posteriormente a su fundación, se inició el auge de la agricultura en la región (florecimiento de la caficultura), empezando la rápida desaparición de los bosques $(15,16)$. Con esta información se puede suponer que la zona fue altamente intervenida desde comienzos del siglo $\mathrm{XX}$.

\section{Densidad de aulladores}

Para este estudio fueron evaluadas 17,7 ha correspondientes al 34,5\% del área total del guadual (Figura 1).En el área evaluada se completó un esfuerzo de muestreo de 350 horas y se identificaron tres grupos de aulladores, con 27 individuos (Tabla 1) y dos machos solitarios (adulto y subadulto) que siempre se observaron juntos. Los individuos solitarios son flotantes en la población y pueden ingresar a otras tropas o ser expulsados de éstas. Por tanto, no se incluyeron en los análisis para calcular la densidad de grupos. Con estos datos se estimó una densidad de 16,9 grupos $/ \mathrm{km}^{2}$ y 163,4 individuos $/ \mathrm{km}^{2}$. El guadual es similar en toda su extensión, por lo que puede tomarse el área muestreada como representativa de este fragmento. Si se extrapola la densidad estimada a toda el área, la abundancia de aulladores para el bosque de Maravélez sería de 84 individuos y 9 grupos. 


\section{Estructura y composición de los grupos de aulladores}

Se identificó la estructura social de los tres grupos residentes en el área evaluada (Tabla 1). El tamaño promedio de las tropas fue de $9 \pm 1$. De esta forma la composición por sexo y edad registrada fue $25,9 \%$ de machos adultos, 29,6 $\%$ de hembras adultas, 7,4 \% de machos subadultos, 3,7\% de hembras subadultas y 33,3 \% de inmaduros. La relación hembras : machos adultos fue de 1: 0,88 y la relación hembras adultas : inmaduros (infantes + juveniles) fue de 1:1,13.

\section{Composición y estructura vegetal}

En las seis parcelas de vegetación se registraron 209 árboles con DAP mayor a $10 \mathrm{~cm}$, distribuidos en nueve especies y cinco familias. La especie más abundante fue la guadua $G$. angustifolia con 189 individuos (90,4\%). Después de la guadua, la familia más abundante fue Moraceae (4,3\%) con tres géneros (Trophis, Ficus y Poulsenia). Las especies del género Ficus (F. yoponensis, F. cf killipii y
Ficus sp.) fueron los de mayor importancia para los aulladores, al igual que las especies Anacardium excelsum (caracolí) de la familia Anacardiaceae y Poulsenia armata (corbón). Estas especies de árboles proporcionan alimento (hojas y frutos) y refugio a los aulladores. Las especies utilizadas por los aulladores sumaron solamente el 7,7 \% del total de árboles encontrados en las parcelas. Con frecuencia los aulladores se alimentaron de una especie de yarumo (Cecropia sp.), la cual no se registró en las parcelas. En los bordes del fragmento algunos de los propietarios acostumbran sembrar guamos (Inga spp.) para atraer a los monos y poder observarlos directamente. Los aulladores usaron la guadua como dormitorio y estructura de desplazamiento, pero no la consumieron.

En la tabla 2 se encuentran los promedios de las variables evaluadas en cada una de las parcelas. Los datos para DAP y área de la copa fueron bajos, principalmente por el predominio de la guadua, que tiende a estandarizar los promedios. Los casos en que se encontró alguna variación, se deben a la presencia de uno o máximo tres grandes árboles de Ficus spp., P. armata y/o A. excelsum que incrementaron

Tabla 1. Tamaño y composición de tres grupos de monos aulladores rojos encontrados en el bosque de Maravélez (La Tebaida, Quindío)

\begin{tabular}{lcccccccc}
\hline & M A & HA & MSA & H S A & M J & H J & I & Total indv \\
\hline Grupo 1 & 2 & 2 & 1 & 1 & 1 & 1 & 2 & 10 \\
Grupo 2 & 3 & 2 & 1 & 0 & 0 & 1 & 2 & 9 \\
Grupo 3 & 2 & 4 & 0 & 0 & 1 & 0 & 1 & 8 \\
Total & 7 & 8 & 2 & 1 & 2 & 2 & 5 & 27 \\
Promedio & 2,33 & 2,67 & 0,67 & 0,33 & 0,67 & 0,67 & 1,67 & 9 \\
\hline
\end{tabular}

M: macho, H: hembra, A: adulto, SA: subadulto, J: juvenil, I: infante

Tabla 2. Variables evaluadas sobre la estructura vegetal en el bosque de Maravélez (La Tebaida, Quindío)

\begin{tabular}{ccccccc}
\hline Parcela & $\begin{array}{c}\text { DAP } \\
(\mathbf{c m})\end{array}$ & $\begin{array}{c}\text { Altura } \\
(\mathbf{m})\end{array}$ & $\begin{array}{c}\text { Área copa } \\
\left(\mathbf{m}^{\mathbf{2}}\right)\end{array}$ & $\begin{array}{c}\text { Cobertura } \\
\text { dosel }(\boldsymbol{\%})\end{array}$ & \# individuos & \# especies \\
\hline 1 & 27,9 & 7,4 & 16,4 & 78,0 & 44 & 2 \\
2 & 20,3 & 7,0 & 19,2 & 73,3 & 24 & 2 \\
3 & 17,9 & 9,0 & 15,6 & 83,2 & 56 & 4 \\
4 & 35,0 & 9,9 & 30,7 & 72,3 & 14 & 4 \\
5 & 18,7 & 7,8 & 14,4 & 81,4 & 19 & 5 \\
6 & 16,0 & 8,7 & 6,8 & 81,5 & 52 & 3 \\
Promedio & 22,6 & 8,3 & 17,2 & 78,3 & 34,8 & 3,3 \\
Sd & 7,32 & 1,1 & 7,8 & 4,6 & 18,0 & 1,2 \\
\hline
\end{tabular}


los promedios. El número de árboles por parcela fue muy alto (alrededor de 35) debido a la gran abundancia de guadua y al poco manejo de este rodal. El número promedio de especies fue muy bajo y varió entre dos y cinco.

\section{Discusión}

La densidad de los aulladores rojos varía ampliamente, fluctuando principalmente alrededor de 35 y $55 \mathrm{indv} / \mathrm{km}^{2}$ en bosques continuos de tierras bajas $(5,17,18)$. Se encuentran pocos estimativos de densidad en bosques de montaña para esta especie. En Colombia, se calculó una densidad de $c a .15 \mathrm{indv} / \mathrm{km}^{2}$ en un bosque no protegido y poco intervenido en el Huila, cordillera Central, a $2300 \mathrm{~m}$ de altitud (19). En Risaralda, en la misma cordillera, a $\sim 1900 \mathrm{~m}$, se reportó una densidad de $73 \mathrm{indv} / \mathrm{km}^{2}$ en un bosque continuo con plantaciones forestales no comerciales y vegetación natural (20). La densidad estimada en Maravélez de 163,4 indiv/ $\mathrm{km}^{2}$ es superior a dichos reportes. Esta alta densidad puede ser un efecto del área pequeña y de su aislamiento (en los alrededores existen pocos y pequeños remanentes de bosque, y pocas posibilidades de comunicación entre éstos), lo cual limita las capacidades de dispersión de los aulladores y los confina en el fragmento $(11,21)$. Densidades altas, con alguna evidencias de alteración de la composición grupal, han sido reportadas en guaduales aislados en Montegrande (Caicedonia, Valle): $255 \mathrm{indv} / \mathrm{Km}^{2}$ (6) y el Ocaso (Quimbaya Quindío): $378 \mathrm{indv} / \mathrm{Km}^{2}$ (8). Para el género Alouatta han sido reportadas densidades menores en bosques continuos comparados con bosques aislados, e incluso se han encontrado fragmentos sobrepoblados $(13,22,23)$. Gracias a su estrategia de ahorro de energía, sus bajos requerimientos de espacio y su dieta folívora facultativa, los aulladores son reconocidos por su capacidad para sobrevivir en ambientes fragmentados, e incluso en cultivos y bosques perturbados $(13,22-26)$.

A pesar de la alta densidad y del aislamiento, las tropas de monos en Maravélez tienen un tamaño y composición similar a las reportadas para la especie $(5,10,17)$. Se encontró un mayor número de inmaduros que de hembras adultas, lo cual indicaría que el número está siendo reemplazado y que la población se está expandiendo (27).

El DAP y la altura son importantes para evaluar el hábitat de los aulladores, puesto que este género de animales son principalmente cuadrúpedos de dosel, que evitan saltar entre árboles. Los aulladores utilizan árboles de gran porte y prefieren los estratos superiores del bosque, con buena continuidad de dosel $(5,10,12,14)$. En las parcelas de vegetación en Maravélez, no se encontró un amplio rango de valores para las variables evaluadas, debido a la abundancia de la guadua. En estos bosques son poco comunes los árboles grandes. Sin embargo, la guadua permite una continuidad en el dosel y ofrece la estructura necesaria para el desplazamiento de estos monos $(5,14)$. Es decir, la guadua presta el esqueleto o soporte para los aulladores, pero no les sirve como fuente de alimento. Las especies vitales para ellos, de las cuales dependen para alimentarse, como las moráceas, particularmente Ficus spp. (24, 28), presentaron una baja cantidad y diversidad. En estudios sobre las respuestas ecológicas de aulladores en la amazonía Brasilera, Venezuela y México, se ha encontrado que grupos que habitan en fragmentos pequeños tienden a alimentarse de un menor número de especies y que explotan menos recursos que los grupos de fragmentos grandes. Se sugiere que la fragmentación y aislamiento afectan negativamente la diversidad vegetal, causando una oferta de recursos limitada para los aulladores $(13,22,24)$.

La población de aulladores de Maravélez está expuesta a algunas amenazas como el efecto negativo del borde (29) sobre los recursos de los monos, puesto que estos guaduales son muy angostos y alargados. También se debe tener en cuenta que los guaduales están embebidos en medio de fincas, con contacto directo con la fauna doméstica, lo cual incrementa la posibilidad de contagio (por ejemplo de la fiebre amarilla y otras virosis trasmitidas por las moscas del ganado, a las cuales son muy susceptibles los aulladores) (25).

Hacia la década de los años 60’s este fragmento presentaba un mínimo de conexión con otros parches de la zona a través de cultivos de sombrío, encontrándose muy cerca de otros guaduales. Sin embargo, en los años 70’s el fragmento ya estaba totalmente aislado. Asumiendo que desde esta década los monos de Maravélez perdieron la posibilidad de dispersarse, se podría hablar de una población de al menos 30 años de aislamiento. Teniendo en cuenta el tamaño tan pequeño del bosque y que se estimó una población de 84 individuos, se puede suponer un alto grado de entrecruzamiento. Mientras más pequeña sea una población, mayor será su probabilidad de extinguirse (2). Por ejemplo, una enfermedad puede propagarse vertiginosamente y acabar con todos los individuos de una misma población, pues en teoría, habría una baja diversidad genética. Estas condiciones, sumadas al estrés causado por la alta densidad, pueden bajar las defensas de los individuos (25). Sin embargo, la población de Maravélez no muestra características de decadencia; los grupos tienen una composición social típica $(5,10,17)$ e incluso se sugiere que está en crecimiento $(11,27)$. Estos resultados indicarían que la población de aulladores de Maravélez no se encuentra en condiciones tan alarmantes de hacina- 
miento y confirma que los aulladores pueden sobrevivir en condiciones extremas como las de estos rodales (22-25, 30). Sin embargo, la pervivencia en esas circunstancias, no implica que la población esté sana y pueda mantenerse en el futuro. No se encuentra información sobre cuánto tiempo pueden soportar las poblaciones de aulladores en condiciones como las de Maravélez y tampoco sobre cuáles son los factores y las variables que indican la cercanía al límite tras el cual se rompe la tolerancia de esta especie a sobrevivir en pequeños relictos, alterados y aislados.

\section{Conclusiones}

El bosque de Maravélez presentó una alta densidad de monos aulladores rojos (163.4 indv/ $\mathrm{km}^{2}$ ), como consecuencia del alto grado de aislamiento y el área pequeña del bosque. A pesar de la alta densidad, las tropas de aulladores presentaron tamaño y composición similar a las encontradas en tierras bajas. En este guadual la diversidad vegetal fue baja, sin embargo se encontraron dispersos, aunque escasos, árboles de importancia para los aulladores como moráceas (principalmente Ficus). La baja abundancia de recursos, el tamaño y aislamiento del bosque, su forma y las actividades antropicas alrededor y dentro del bosque son posibles amenazas para la persistencia de esta población a futuro. Estos resultados demuestran la tolerancia de esta especie a la fragmentación y a la pérdida de diversidad vegetal. Sin embargo, esta población de aulladores se encuentra aislada de otras poblaciones y por tanto es urgente desarrollar medidas de manejo del paisaje para atenuar los efectos del aislamiento y que incrementen la posibilidad de conexión con otras poblaciones cercanas de aulladores.

\section{Agradecimientos}

Los autores agradecen a los propietarios del bosque en el Valle de Maravélez, especialmente a Mario Gutiérrez y familia (Finca La Cascada) por el apoyo logístico y el permiso para trabajar en sus predios. A Jorge Mario Londoño por el apoyo logístico. A Leonor Valenzuela por la ayuda con la georeferencición de las fotografías aéreas. Al Dr. Gustavo Kattan por su continuo apoyo al proyecto.

\section{Financiación}

Este trabajo fue financiado por la Fundación para la Promoción de la Investigación y la Tecnología del Banco de la República (proyecto 1.758), John D. and Catherine T. MacArthur Foundation, e Idea Wild

\section{Conflicto de intereses}

Los autores afirman no tener conflictos de intereses.

\section{Referencias}

1. Etter A, Villa A. Andean forest and farming systems in part of the eastern cordillera (Colombia). Mt Resource Development 2000; 20: 236-243.

2. Kattan G, Álvarez-López H. Preservation and management of biodiversity in fragmented landscape in the Colombian Andes. En: Schelhas J, Greenberg R. (eds.). Forest patches in tropical landscape. Island Press. Washington D.C., USA. 1996; 3-18.

3. Ospina R, Finegan B. Variabilidad florística y estructural de los bosques dominados por Guadua angustifolia en el Eje Cafetero colombiano. Recursos Naturales y Ambientes 2004; 41: 25- 33.

4. Cataño F, Villanueva G, Soto F. Evaluación del desarrollo de los bosques de Guadua angustifolia en la zona de la jurisdicción de la CVC bajo diferentes condiciones de sitio, con fines de reforestación. Corporación Regional del Valle del Cauca CVC. Cali, Colombia. 2002, 23 p.

5. Defler T. Primates de Colombia. Serie de Guías Tropicales 4. Conservación Internacional. Bogotá, D.C., Colombia. 2003, 543 p.

6. Gómez-Posada C, Roncancio N, Hincapié P. Betancourt A. En imprenta. Densidad poblacional y composición de grupos en tres poblaciones de mono aullador rojo en Cauca y Valle del Cauca. Boletín Científico del Centro de Museos, Universidad de Caldas.

7. Gómez-Posada C. Biología y estado de conservación del mono aullador rojo. En: Kattan G, Valderrama C. (eds.). Plan de conservación del mono aullador rojo (Alouatta seniculus) en la región del Sirap Eje Cafetero y Valle del Cauca. Instituto de Investigación de Recursos Biológicos Alexander von Humboldt y WCS Programa Colombia / Fundación EcoAndina. Bogotá, D.C., Colombia. 2006; 13-40.

8. Gómez-Posada C, Londoño JM. Evaluación del estado de la población del mono aullador rojo (Alouatta seniculus) en la Reserva la Montaña del Ocaso. Fundación EcoAndina / WCS Colombia. Cali, Colombia. 2007, 50 p. 
9. Plan de Ordenamiento Territorial 1999-2006. Municipio de La Tebaida. Departamento del Quindío. Armenia, Colombia. 1999, 95 p.

10. Soini P. Ecología del coto mono (Alouatta seniculus, CEBIDAE) en el río Pacaya, Reserva Pacaya - Saimiria, Perú. Folia Amazónica 1992; 4: 103-118.

11. Defler T. The density of Alouatta seniculus in the eastern llanos of Colombia. Primates 1981; 22: 564-569.

12. Stevenson P, Quiñones M, Ahumada J. Influence of fruit availability on ecological overlap among four neotropical primates at Tinigua National Park, Colombia. Biotropica 2000; 32 (3): 533-544.

13. Estrada A, Coates-Estrada R. Tropical rain forest fragmentation and wild populations of primates at Los Tuxtlas, Mexico. International Journal of Primatology 1996; 17: 759-783.

14. Yoneda M. Habitat utilization of six species of monkeys in Río Duda, Colombia. Field Studies in New World Monkeys in La Macarena, Colombia 1988; 1: 39-45.

15. Valencia A. La guaquería en el viejo Caldas. Biblioteca Virtual Luís Ángel Arango. Bogotá, D.C., Colombia. 2005. http://www.lablaa.org/blaavirtual / publicacionesbanrep/bolmuseo /1989/bol23/boj2.htm. Revisado febrero de 2006.

16. Centro de Investigaciones y Educación Popular CINEP. Colombia país de regiones. Tomo I. Biblioteca Virtual Luís Ángel Arango. Bogotá, D.C., Colombia. 2004. 'http://www.lablaa.org /blaavirtual/geografi a/ region1/indice.htm. Revisado febrero de 2006.

17. Chapman C, Balcomb S. Population characteristics of howlers: ecological conditions or group history. International Journal of Primatology 1998; 19: 385403.

18. Crockett C, Eisenberg J. Howlers: variations in group size and demography. En: Smuts B, Cheney D, Seyfarth R, Wrangham R, Struhsaker T. (eds.). Primate Societies. The University of Chicago Press. Chicago, USA. 1987; 54-68.

19. Gaulin S, Gaulin C. Behavioral ecology of Alouatta seniculus in Andean cloud forest, Colombia. International Journal of Primatology 1982; 3: 1-32.

20. Gómez-Posada C, Martínez J, Giraldo P, Kattan G. Density, habitat use and ranging patterns of red howler monkey in Andean forest. Neotropical Primates 2007; 14 (1): 2-10.

21. Pruetz Jd, Leasor Hc. Survey of three primate species in forest fragments at La Suerte Biological Field Station, Costa Rica. Neotropical Primates 2002; 10: 4-9.

22. Orihuela-López G, Terborgh J, Ceballos N. Food selection by a hyperdense population of red howler monkeys (Alouatta seniculus). Journal of Tropical Ecology 2005; 21: 445-450.

23. Rodríguez-Toledo EM, Mandujano S, García-Orduña F. Relationships between forest fragments and howler monkeys (Alouatta palliata mexicana) in southern Veracruz, México. En: Marsh LK (ed.). Primates in fragments: ecology and conservation. Kluwer Academic / Plenum Publisher. New York. USA. 2003; 79$97 \mathrm{p}$.

24. Bicca-Marques JC. How do the howlers cope with habitat fragmentation?. En: Marsh LK (ed.). Primates in fragments: ecology and conservation. Kluwer Academic / Plenum Publisher. New York. USA. 2003; p. 283-304.

25. Crockett C. Conservation biology of the Genus Alouatta. International Journal of Primatology 1998; 19: 549-579.

26. Milton K. The foraging strategy of howler monkeys. A study in primate economics. Columbia University Press. New York, US. 1980, 165 p.

27. Heltne PG, Turner DC, Scott Jr NC. 1976. Comparison of census on Alouatta palliata from Costa Rica and Panamá. En: Thorington R, Heltne PG. (eds.). Neotropical Primates: field studies and conservation. National Academy of Science. Washington, D.C., USA. 1966; 0-19.

28. Giraldo P, Gómez-Posada C, Martínez J, Kattan G. Resource use and seed dispersal by red howler monkey (Alouatta seniculus) in Andean Forest. Neotropical Primates 2007; 14: 55-64.

29. Kattan G. Fragmentación: patrones y mecanismos de extinción de especies. En: Guariguata MR, Kattan GH. (eds.). Ecología y conservación de bosques neotropicales. Libro Universitario Regional. Cartago, Costa Rica. 2002; 561-590.

30. Rylands A, Keuroghlian A. Primate populations in continuous forest and forest fragments in Central Amazonia. Acta Amazonica 1988; 18: 291-307. 